\title{
Transthoracic ultrasound for pleural effusion: traps and tricks
}

\author{
Marco Sperandeo ${ }^{1}$ \\ Daniela Catalano ${ }^{2}$ \\ Nadia Melillo 3 \\ Tiziana Foti ${ }^{4}$ \\ Guglielmo M. Trovato ${ }^{2}$
}

\author{
1 IRCCS "Casa Sollievo della Sofferenza" Hospital - \\ Section of Interventional Ultrasound of Internal Me- \\ dicine, San Giovanni Rotondo (FG), Italy \\ 2 Department of Medical and Pediatric Sciences, \\ Postgraduate School of Clinical Echography, Univer- \\ sity of Catania, "Azienda Policlinico-Ospedale Vittorio \\ Emanuele", Catania, Italy \\ ${ }^{3}$ Department of Rheumatology, University of Foggia, \\ Foggia, Italy \\ 4 Pulmonary Occupational Disease Unit, Termoli (CB), \\ Italy
}

Address for correspondence:

Guglielmo M. Trovato, MD

Department of Medical and Pediatric Sciences

Policlinico Hospital, University of Catania,

Via Santa Sofia - Catania, Italy

Phone: +390953781533

Fax: +390953781549

E-mail: guglielmotrovato@unict.it

\section{Summary}

Pleural effusion is excess fluid that accumulates between the two pleural layers. Excessive amounts of such fluid can impair breathing by limiting the expansion of the lungs during ventilation.

Pleural effusion is still usually diagnosed on the basis of medical history and physical examination, and confirmed by chest x-ray. It is observed in many pulmonary and extra-pulmonary disease; its cause can be relatively benign or definitely malign (cancer), and may require drainage for treatment or for achieving a diagnosis which is available at the bedside by physical examination and Thoracic Ultrasound (TUS). Pleural effusion is detected by TUS even when its volume is very little: it is possible to perform and repeat at bedside, by sufficiently trained physicians. Diagnostic intervention procedures are safer if performed using US probes specifically designed for this use, i.e. with a central hole which allows the co-axial passage of the disposable tools for drainage or Fine
Needle Aspiration Biopsy (FNAB). Over-trusting in US criteria not evidence-based and, more important, which are demonstrated to be unreliable when critically re-appraised must be discouraged; reversal of such scarcely validated but recommended practices, which could be harmful for patients, is actively in progress also in this field of medicine. No special trick is needed and no actual trap is present when the assessment of pleural effusion is performed by a sufficiently skilled MD and with a reliable and well set echo machine. Echo-assisted thoracentesis is an excellent procedure when appropriately performed in all its phases, which are: choice of the site of insertion, visualization in real-time during the drainage and serial control during lung re-expansion (so as to avoid pneumothorax).

KEY WORDS: ultrasound, pleural effusion.

Transthoracic ultrasound: overview

The ultrasound examination in the study of pleuro-pulmonary disease, i.e. Thoracic Ultra-Sound (TUS), is limited by the presence of air in the lungs and by the rib cage (1). As a consequence

TUS is not an all purpose tool, drawbacks and appropriate indications must be the cynosure of the pneumologist which relies on TUS for diagnosis and management of patients (2). The presence of the bony structures of the rib cage restricts the success of ultrasound imaging of the pleural
Pleuro-pulmonary TUS can be a key aid for the guidance of interventional diagnostic methods in the field of pulmonology, such as pleural and lung biopsies and pleural drainage. surface to about $70 \%$ of the total (3). These issues, relating to limitations on the use of ultrasound in the thoracic area, have been known since the '60s, a time when pioneering works were published using A-mode for exploring pleuro-pulmonary structures and, namely, pleural effusion (4), even in comparison with radiological imaging and with a greater sensitivity and specificity (5). Thereafter these advantages were established in the few subsequent years, with minimal further refinements despite the technology of ultrasound equipment has evolved considerably $(6,7)$ : the value of ultrasound examination of the pleura and lungs remains highly underestimated to this day, and overstatements (8) and over- 
confidence in unreliable criteria (9-12) is increasing the distrust in TUS, undermining the actual credit of this procedure.

Despite these limitations, ultrasound has gone, over time, more and more emerging as a reliable, cheap, harmless and repeatable tool, valuable for the study of diseases limited to the diaphragm (mainly the right part), chest wall (abscesses, fistulas, tumors), the mantle zone of the lung (atelectasis, pulmonary consolidations and space-occupying peripheral lesions), the anterior superior mediastinum (tumors, lymphomas, cysts), and, overall, defined by the thoracoabdominal border area; above all, pleura (including the extra-pleural masses) and pleural effusions are the most appropriate targets of TUS assessment and US guided intervention procedure, such as thoracenthesis (13) and FNAB (14).

TUS is always complementary to the thoracic radiographic study (Chest X-Rays, i.e. CXR) and/or to chest CT scan $(15,16)$, and therefore should never be considered end point of a diagnostic process and workup (17).

Pleuro-pulmonary TUS can be a key aid for the guidance of interventional diagnostic methods in the field of pulmonology, such as pleural and lung biopsies and pleural drainage $(13,14)$; both procedures are even less invasive due to the use of probes with a central passage of the fine needle devices, which allow the best approach to interventional pulmonology. Pleuropulmonary TUS is being increasingly used in Emergency Departments, in all those cases in which such additional examination is a useful guide for the subsequent and immediate diagnostic approach for further study, which are always complementary to chest physical examination and to other established imaging modalities (16).

\section{Equipment, methods and physics of ultrasound}

Chest Sonography should be performed with ultrasound machines equipped with medium frequency convex or sector probes from 3.5-5.0 MHz; also linear, high-frequency $(8-12.5 \mathrm{MHz})$ probes are used in the study of the chest wall, pleura and superficial structures. The 2-8 MHz multifrequency probes, which can be considered the more recent development, are use-

The power and color Doppler become of fundamental help during interventional chest US guided procedures, including thoracentesis in pleural effusion, since they allow the view of the vessels in the superficial tissues of the passage through the rib cage. ful for a preliminary diagnostic and, often, even for a conclusive report. The application (preset) for the chest is not normally preinstalled in any ultrasound system and therefore it is necessary to start and save in memory a preset for the ultrasound pleuro- pulmonary assessment; this step is necessary for both the convex and the linear probes. This installation requires a specific knowledge of all the features of the machine as well as a good knowledge of the physics of ultrasound. For these procedures, please refer to insights and further information in texts devoted to this topic (16-18).

Color and pulsed Doppler are not critical for a first approach ultrasound examination, because are both very often affected by respiratory motion artifacts and heart rate, flash artifacts, for example (19), even optimizing the setting (PFR). The power- and colorDoppler become of fundamental help during interventional chest US guided procedures, including thoracentesis in pleural effusion, since they allow the view of the vessels in the superficial tissues of the passage through the rib cage (the vessels of the chest wall).

No special preparation of the patient before the examination is needed; in cases of severe respiratory distress, patients should be treated pharmacologically in advance and oxygen should be provided during the examination. This clinical approach will allow symptoms relief and prevent large thorax excursion that increase motion artifacts. Not infrequently, in fact, the examination must be performed at the bedside, for severe medical conditions.

TUS examination should be carried putting initially the patient in a sitting position, and then, when possible, with the patient in the supine position, in order to assess the basal pleura and the diaphragm with its movement, by sub-costal scans pointing initially on the liver and spleen. In special cases, the orthostatic position can be used for an accurate assessment of small fluid effusions at the level of the costal-phrenic sinuses $(20,21)$

Scans to be carried out are many. From the back, posteriorly, we will use intercostal chest scans: longitudinal, transverse and paravertebral. Furthermore, anterior intercostal, longitudinal and transverse scans are used, and, at last, parasternal, subxyfoid and supraclavicular. For a better visualization of the structures in the basal posterior and lateral view, it is useful to get adequate acoustic windows right from the liver. For the evaluation of the diaphragm is more suitable the supine position, during forced inspiration, in order to evaluate the basal pleura and the diaphragmatic dome. Other thoracic findings can be searched in a targeted manner on the basis of any standard CXR or chest CT scan already performed. The abnormal findings must be rather documented in two views, perpendicular to each other. Below the pulmonary pleura the presence of air will spread out completely the ultrasound beam. In fact, in correspondence of the pleural line, the point of passage and site of the high acoustic difference between the soft tissue surface and the pulmonary air, there is a reflection $>96 \%$ of the echoes transmitted by the probe.

The pleural line is hyperechoic and moving with respi- 


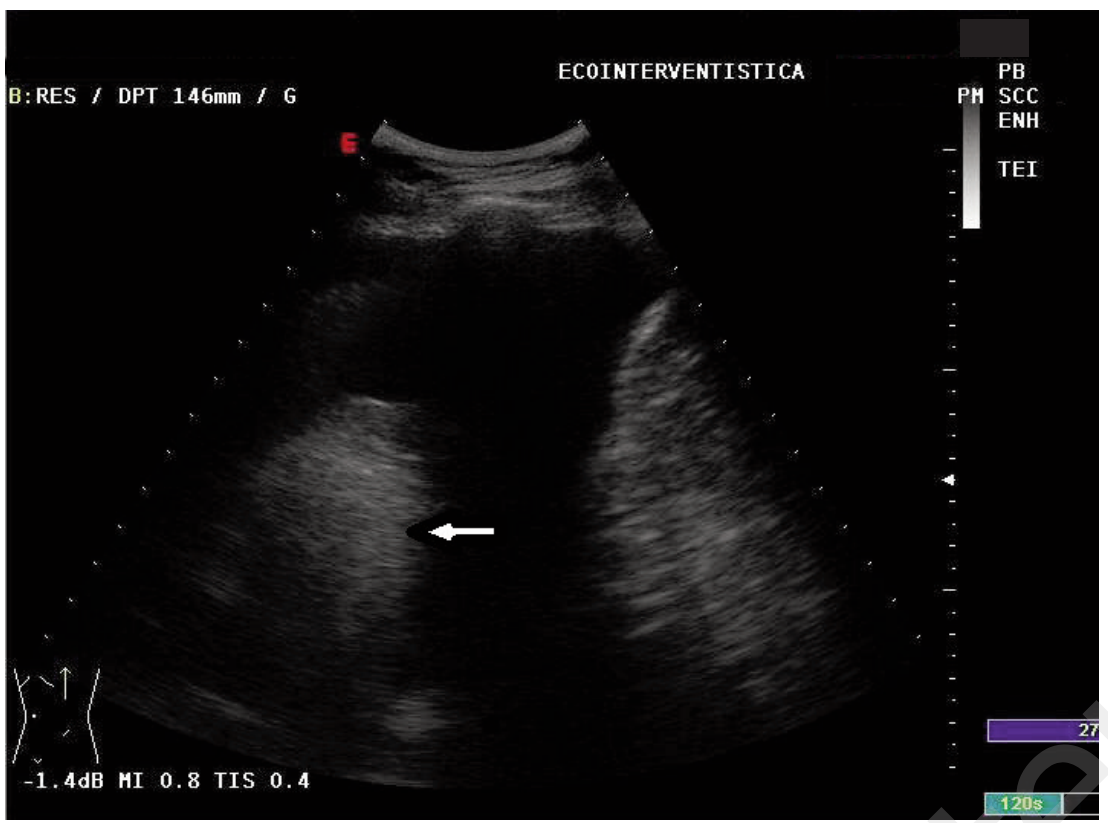

Figure 1 - Anechoic pleural effusion in posterior-basal longitudinal view by a convex $3.5 \mathrm{MHz}$ probe; note, please, the concurrent atelectasis (Arrow).

ratory excursions, due to pleural membrane gliding $(22,23)$ or sliding, i.e. jointly slipping below the chest cage (16); its measure under normal conditions is approximately $2 \mathrm{~mm}(1.4-2.6 \mathrm{~mm})$ when using the convex probe 3.5-5 $\mathrm{MHz}$ and 0.8-1.6 $\mathrm{mm}$ when using the 8-12.5 MHz linear transducer. The pleural line is not the only expression of anatomical pleura and of the pleural space, (which would measure a maximum of 300 microns), but rather appears to be the result of the physical effect by which ultrasound, due to the high difference in acoustic impedance between the superficial soft tissues (skin, subcutaneous tissue, intercostal muscles, endothoracic fascia, fat, and pleura) and the air in the lung, i.e. for a soft tissue/air interface $96 \%$ is reflected.

Such a large difference in acoustic impedance also creates artifactual images normally present in healthy subjects and defined reverberation artifacts: 1) the simple reverberations or lines $\mathrm{A}$, are hyperechoic horizontal lines arranged in concentric bands below the pleural line; 2) the "comet tail" a few millimeters long (3-4 $\mathrm{mm}$ ) and typically with a triangular shape (hence the name "comet tail") and 3) the "ring-down "or B lines, or hyperechoic vertical lines, perpendicular to the pleural line and mobile with respiratory excursions, not triangular and with much longer tail than the comet. All these artifacts are usually and commonly present, to a lesser extent, even in normally aerated lung and are not pathological (24).

These artifacts are also present even in the pneumonectomy space of patients undergoing pneumonectomy, according with the physical principles of ultrasounds, which are generated by the large difference in acoustic impedance between the superficial soft tissues of the rib cage and the air and fluid in the residual cavity after pneumonectomy (25).

\section{Diagnostic ultrasound in pleural effusions}

Chest ultrasonography is the "gold standard" method for the study of pleural effusions (26).

Ultrasound diagnosis of pleural effusion is simple and accurate. The identification of a pleural effusion is also possible for small amounts of liquid (below $10 \mathrm{ml}$ ), and therefore with a diagnostic yield far superior to standard chest radiography or other imaging methods. The
Ultrasound also allows to determine the content of the effusion and then a diagnosis of the nature of the effusion itself. effusion will appear as an anechoic image, with welldefined margins, with a shift in the depth of the pleural line and below it you can see the ventilated lung (in pleural effusions thins and uniformly extended throughout the lung) or, if any, consolidated or atelectasic lung areas (Figure 1). Some authors have developed formulas for calculating the volume of the effusion (27), the amount of compression-related lung atelectasis and the degree of organization of the pleural fluid. Although some authors have tried to calculate it, the quantification of the effusion by ultrasound is not really reliable since the method is two-dimensional and any calculation formula cannot provide a safe and reliable quantification of three-dimensional real volume and quantity (16). In clinical practice, ultrasound, however, may be sufficient to determine the greater longitudinal and transverse diameters of the effusion (Figure 2). This would allow, in any case, an objective assessment for any follow-up throughout medical treatment or after drainage.

Ultrasound also allows to determine the content of pleural effusion, so providing a possible diagnosis of the nature of the effusion. Characteristically, we distin- 


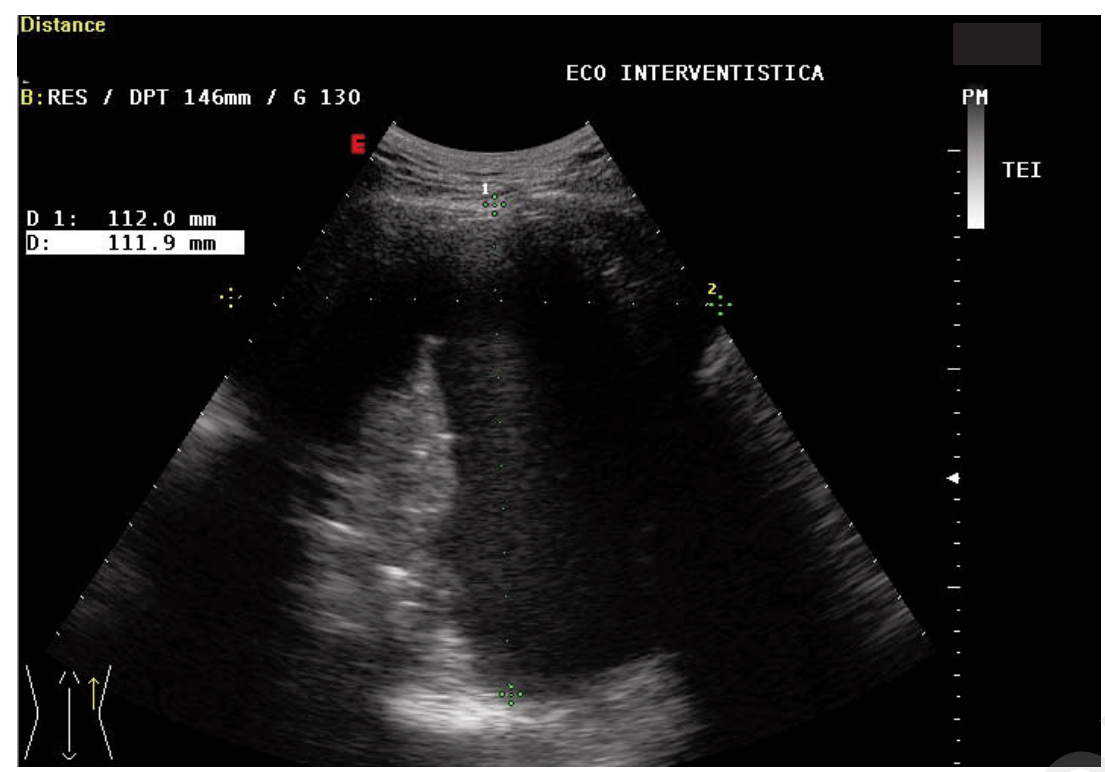

Figure 2 - Right Basal pleural effusion, measured by two orthogonal views (Longitudinal and transversal), viewed by longitudinal scan using a convex $3.5 \mathrm{MHz}$ probe.

guish four patterns of pleural effusion: 1) anechoic; 2) complex not septated; 3) complex septated; 4) homogeneously hyperechoic (28) as shown in Figure 3 A-D.
It is also possible to highlight the presence or absence of fibrous transformation (presence of fibrinous septa, or loculated pleural effusion), it is not uncommon, in

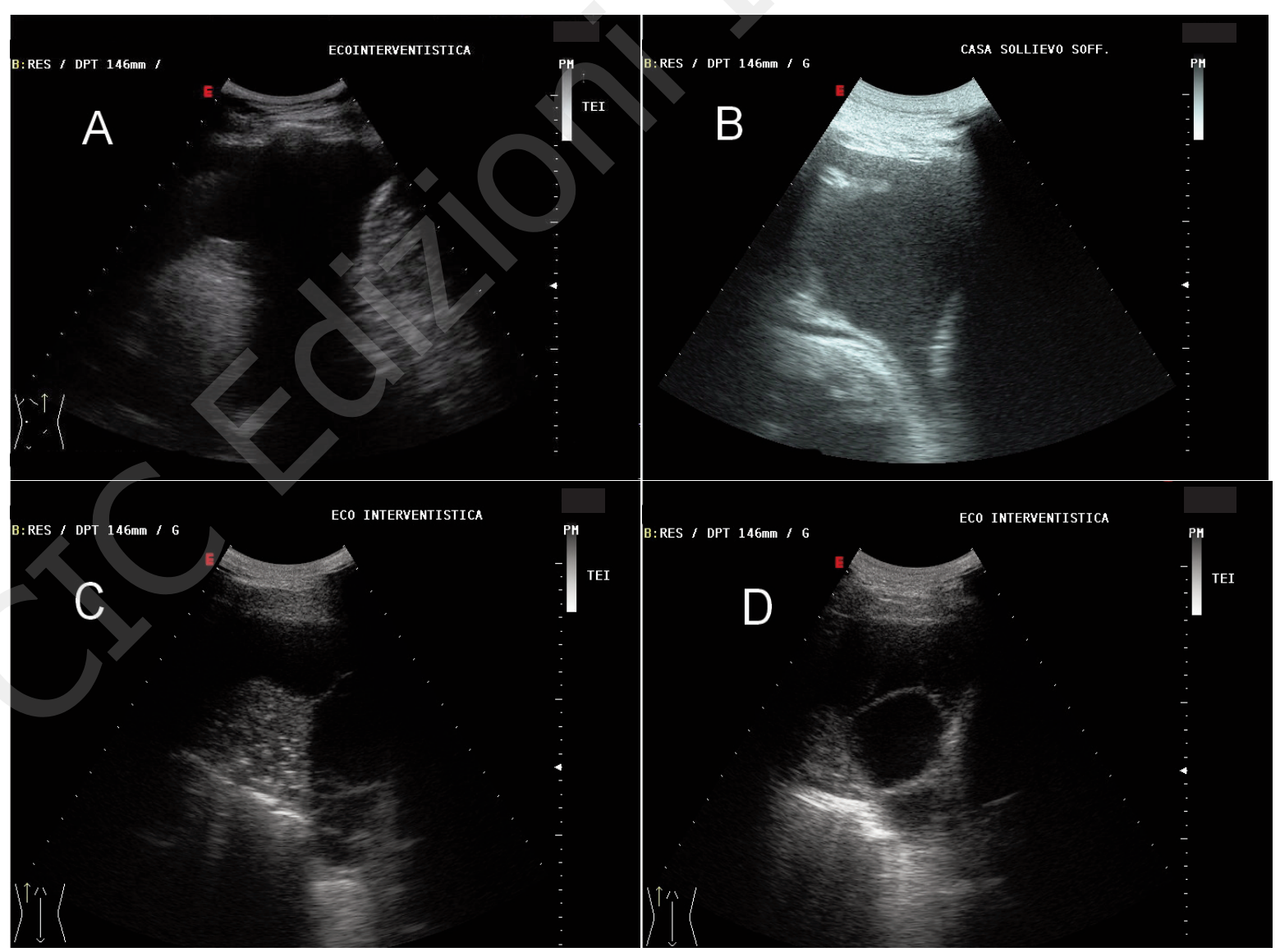

Figure 3 A-D - A) anechoic pleural effusion; B) homogenous hyperechoic pleural effusion; C) complex, non loculated pleural effusion; D) loculated pleural effusion. 


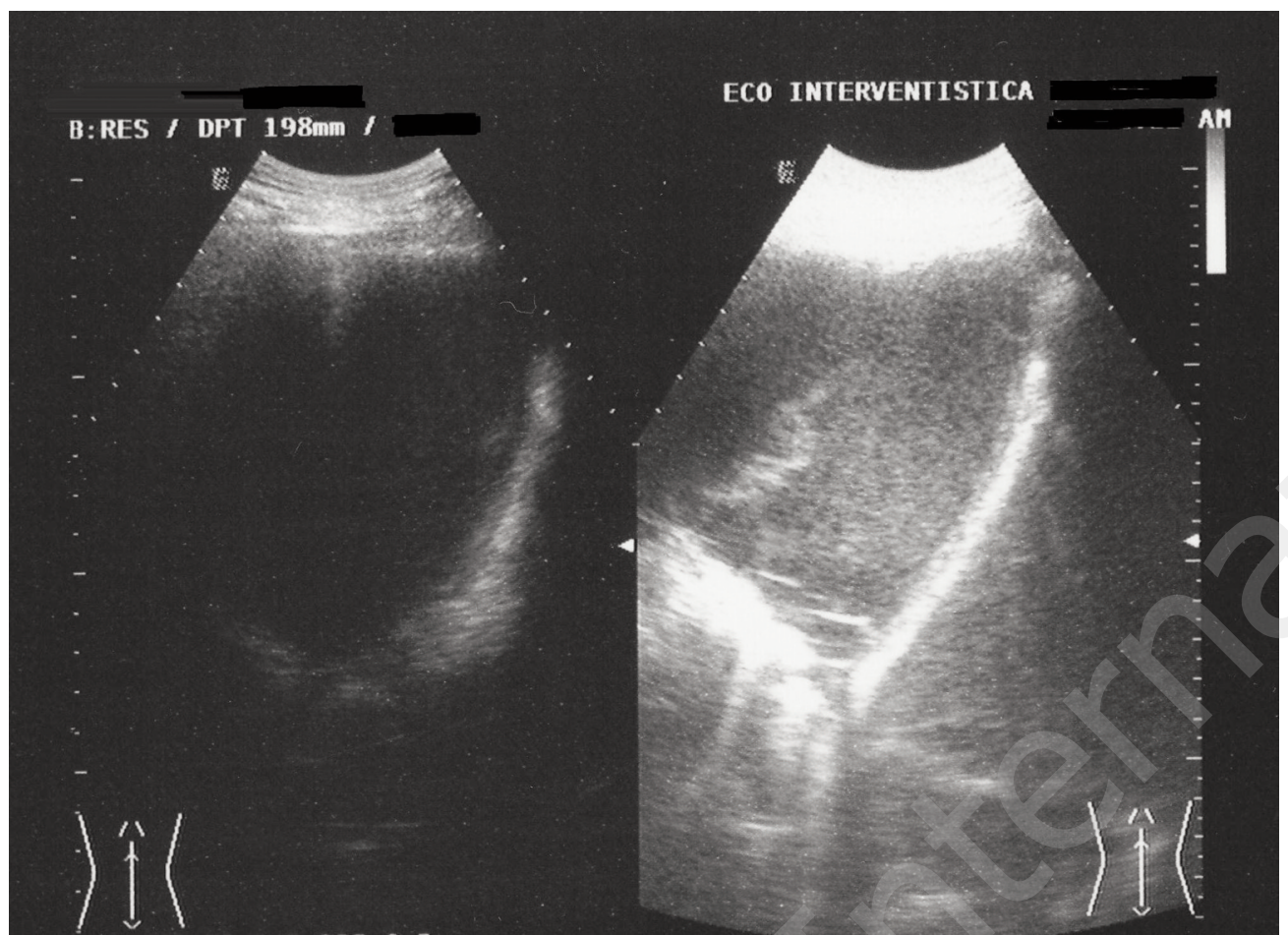

Figure 4 - Longitudinal basal scan showing a pleural effusion: right, excessive gain which address to an fake hyperechoic effusion, suggesting corpuscular elements, while on the right the correct gain displays correctly an anechoic pleural effusion.

the hardening process, called organization, of pleural effusions a concurrent pleural thickening; the occurrence of echoes within the liquid may point to corpusculated effusions with high protein concentration (exudates), empyema (when do not modify with postural changes), hemothorax, malignant effusions (which are often associated with a fringed appearance and nodular thickening of pleural membranes), which are all, however, confirmed by thoracentesis. In this regard, it is vital to remember the importance of the optimal setting of the ultrasound equipment. In fact, a gain curve adjusted in excess (increased gain) make the appearance of the effusion inevitably hyperechoic and then falsely corpusculated (Figure 4); this could lead to erroneous management approaches, such as the choice of needles for thoracentesis of a gauge larger than needed.

The free fluid moves varying according to the decubitus of the patient, unlike the loculated effusion, which is much less modifiable, and differently from empyema, which does not change with the body position; the latter is easily distinguishable from fibrothorax which appears as a band of variable echogenicity, sometimes calcified, parallel to the coast, of uniform thickness, which does not modify with breath changes. Also sub-pulmonary effusions may be visible, if adjacent to the pleura, often in association with inflammatory or cancer processes (29). In addition, ultrasound can detect by trans-thoracic anterior left view the presence of any posterior pericardial effusion. A fundamental application of ultrasonography in pleural effusions is the ability to guide the needle in thoracentesis performed for diagnostic and/or therapeutic purpose, without any risk of complication, being able to drain deposits also of negligible size and volume, as those of the costalphrenic angle places, using the sitting position which allow to evaluate small effusion volumes (Figure 5) up to $10 \mathrm{ml}(30)$.

\section{Interventional ultrasound-guided procedures in pleural effusions}

Ultrasound offers the opportunity to guide the needle thoracentesis if the effusion is more significant and abundant (Figure 6), as well as to avoid, during the lung re-expansion throughout and after drainage, the risk of pneumothorax $(30,31)$. Also, since the ultrasound allows to make a diagnosis of the nature of the effusion (simple, loculated, organized), as well as to better distinguish the clouding visible to the chest $\mathrm{x}$-ray (for example, to distinguish an effusion from a dully baseline pulmonary consolidation ) $(13,14,16)$, it is possible to decide in advance which gauge needle must be used (20 $\mathrm{G}$ if the effusion is anechoic, $18 \mathrm{G}$ if the effusion is corpusculated). As elsewhere reported, the percentage of pneumothorax without the assistance

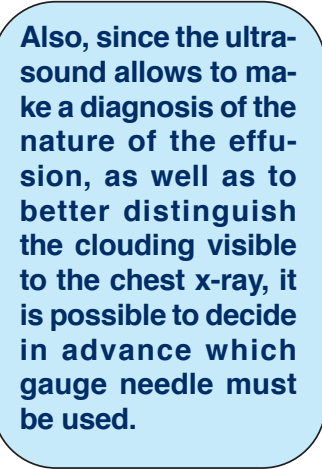




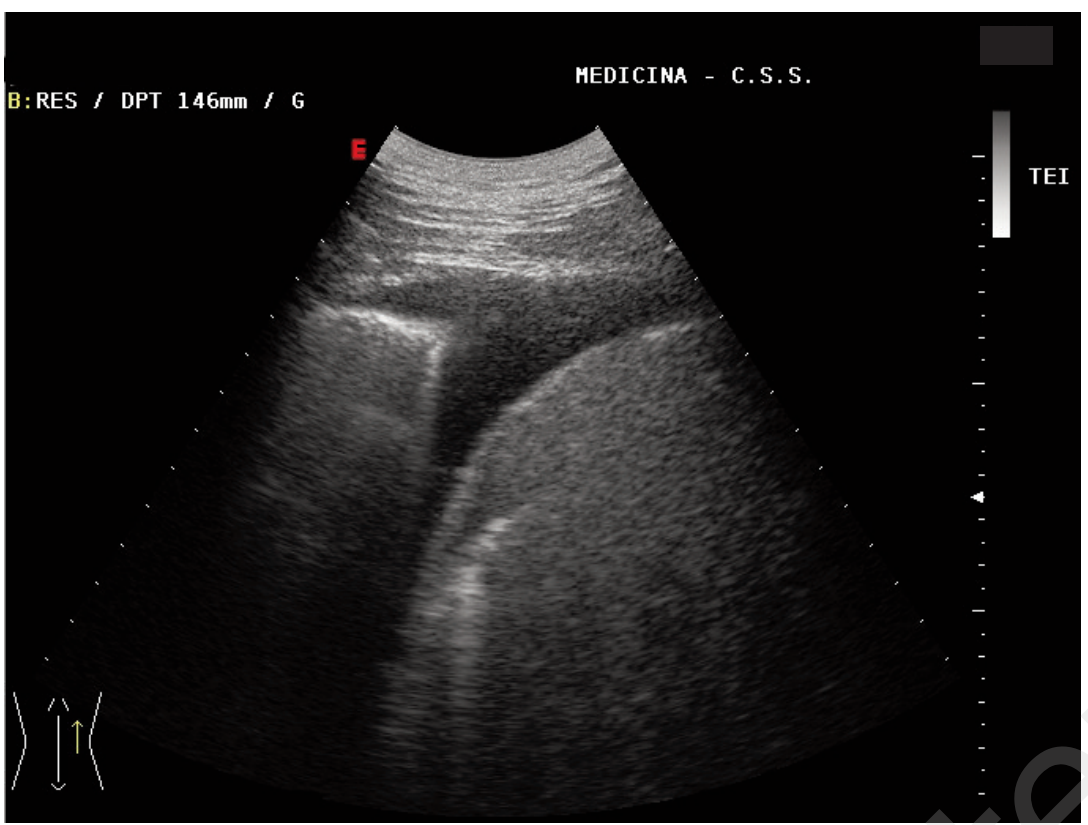

Figure 5 - Small pleural effusion of the right costodiaphragmatic recess detected by a convex probe through longitudinal right scan.

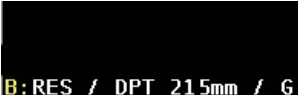

CASA SOLLIEVO SOFF.

B: RES

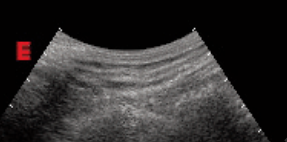

$\mathrm{AGO}$

\section{SOFF.}
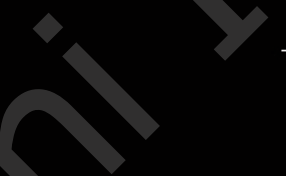

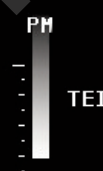

TEI
Figure 6 - Basal pleural effusion showing the tip of the needle (hyperechoic spot) within the fluid during thoracentesis. of ultrasound is estimated from 7 to $15 \%$, which falls to $0.5 \%$ if assisted by the ultrasound (13).

In our thoracentesis case series performed throughout a 10 years period, we performed 3870 consecutive drainages (diagnostic and therapeutic) under ultrasound assistance. A diagnosis of malignant effusion was done in 630 patients $(18 \%)$, by the detection of neoplastic cells at the cytological examination of the drained fluid. The rate of major complications was low: only four out of the patients $(0.1 \%)$ had iatrogenic pneumothorax: 3 patients showed partial pneumothorax and 1 patient subtotal pneumothorax, with full lung re-expansion in each case. In 2 cases there was transient pulmonary edema due to re-expansion of the contra-lateral lung (13).

A particular remarkable usefulness of ultrasound equipment is in the Emergency Department, where in case of acute respiratory illness, ultrasound examination allow to check and confirm the clinical suspicion of massive pleural effusion and then it consent to drain pleura under echo-guidance, in order to reduce the risk of pneumothorax near to zero. In addition, using a vacuum aspiration device with low flow and low pressure it is possible to drain massive pleural effu- 
sions in a reasonably brief time and with small gauge needle $(20 \mathrm{G})$ also in critically ill patients (21).

Typically in patients with lung cancer, where effusion is concurrently present, thoracentesis may be the first approach to make a diagnosis. Evacuative therapeutic drainage in cancer patients after thoracentesis usually does not prevent that the effusion forms again in a short time, so it is more appropriate to insert a drainage thoracostomy tube. Furthermore, since progressive neoplastic effusions arise slowly, the cause of any dyspnea in cancer patients must be investigated easily by TUS trying to detect the presence of pleural effusion. Less commonly, a diagnostic thoracentesis is needed in case of small effusions secondary to pneumonitis, given the usual absence of bacteria (sterility).

\section{Final recommendation}

Echo-assisted thoracentesis is an excellent procedure when appropriately performed in all its phases, which are:

A) choice of the site of insertion

B) visualization in real-time during the drainage

C) serial control during lung re-expansion (so as to avoid pneumothorax).

Also the use of a dedicated probe (hole in the center) allows the accurate insertion of the needle also in effusions of small size.

There is no actual trick, but adequate training and knowledge of the imaging and intervention procedure are mandatory. The facility of adequate disposable sets for drainage is the counterpart of the easy-to-get

The traps, in these conditions, are not many and the only risky trap is, as above addressed, overtrusting in US criteria not evidence-based and, more important, which are demonstrated to be unreliable when critically reappraised.

US machines, equipped with reliable and suitable convex and linear probes, and set appropriately for superficial and lung imaging.

The traps, in these conditions, are not many and the only risky trap is, as above addressed, over-trusting in US criteria not evidencebased and, more important, which are demonstrated to be unreliable when critically re-appraised; reversal of such scarcely validated but recommended practices, which could be harmful for patients, is actively in progress $(32,33)$ also in this field of medicine.

\section{KEYNOTES}

\section{PLEURAL EFFUSION - WHAT}

Pleural effusion is an excess of fluid that accumulates between the two pleural layers. Excessive amounts of pleural fluid can impair breathing by limiting the expansion of the lungs during ventilation. Pleural effusion is still usually diagnosed on the basis of medical history and physical examination, and confirmed by chest $x$-ray. Chest x-ray acquired in the lateral prone position (with the patient lying on his side) are more sensitive and can pick up as little as $50 \mathrm{ml}$ of fluid. At least $300 \mathrm{ml}$ of fluid must be present before upright chest films can pick up signs of pleural effusion (e.g., blunted costophrenic angles).

\section{PLEURAL EFFUSION - WHEN AND WHY}

Pleural effusion is observed in many pulmonary and extra-pulmonary disease. Its cause can be relatively benign or definitely malign (cancer), and may require drainage for treatment or for achieving a diagnosis.

It requires a timely and reliable diagnosis which is available at the bedside by physical examination and Thoracic Ultrasound (TUS).

\section{PLEURAL EFFUSION DRAINAGE - WHERE AND HOW}

Pleural effusion may be detected by TUS even when its volume is very small: the manoeuvre is simple and repeatable at bedside, by trained physicians.

Diagnostic intervention procedures are safer when performed with US probes specifically designed for this use, i.e. with a central hole which allows the coaxial passage of the disposable tools for drainage or Fine Needle Aspiration Biopsy (FNAB).

\section{NO TRICK, PLEASE, NO TRAP}

No special trick is needed and no actual trap is present when the assessment of pleural effusion is performed by a sufficiently skilled physician using a reliable and properly set echo machine.

"Tricks and treachery are the practice of fools, that don't have brains enough to be honest" (Benjamin Franklin)

"Build a better mousetrap and the world will beat a path to your door" (Ralph Waldo Emerson)

\section{References}

1. Dunn F, Fry WJ. Ultrasonic absorption and reflection by lung tissue. Phys Med Biol. 1961 Apr;5:401-10.

2. Sperandeo, M. Ecografia del torace. Testo atlante. Cic Edizioni Internazionali, Milano, 2004.

3. Di Marco V, Ginardi V, Mazzone O, Trovato GM. Acute pericarditis and pleuritis during maintenance hemodialysis. G Ital Cardiol. 1980;10(1):79-83.

4. Joyner CR Jr, Herman RJ, Reid JM. Reflected ultrasound in the detection and localization of pleural effusion. JAMA. 1967;200(5):399-402.

5. Gryminski J, Krakówka P, Lypacewicz G. The diagnosis of pleural effusion by ultrasonic and radiologic techniques. Chest. 1976;70(1):33-7.

6. Sperandeo M, Sperandeo G, Varriale A. Utilità dell'ecografia nello studio della patologia toracica. Giorn Ital Ultrason. 1991;2(1):9-21.

7. Dietrich CF, Hirche TO, Schreiber D, Wagner TO. Sonographie von Pleura und Lunge. Ultraschall Med. 2003;24(5):303-11.

8. Trovato GM, Catalano D, Martines GF, Sperandeo M. 
Is it time to measure lung water by ultrasound? Intensive Care Med. 2013;39(9):1662.

9. Trovato GM, Sperandeo M. Sounds, ultrasounds, and artifacts: which clinical role for lung imaging? Am J Respir Crit Care Med. 2013;187(7):780-1.

10. Sperandeo M, Trovato GM, Catalano D. Quantifying B-lines on lung sonography: insufficient evidence as an objective, constructive, and educational tool. J UItrasound Med. 2014;33(2):362-5.

11. Trovato GM, Catalano D, Sperandeo M. Echocardiographic and lung ultrasound characteristics in ambulatory patients with dyspnea or prior heart failure. Echocardiography. 2014;31(3):406-7.

12. Trovato GM, Rollo VC, Martines GF, Catalano D, Trovato FM, Sperandeo M. Thoracic ultrasound in the differential diagnosis of severe dyspnea: a reappraisal. Int J Cardiol. 2013;167(3):1081-3.

13. Trovato GM, Sperandeo M, Catalano D. Thoracic ultrasound guidance for access to pleural, peritoneal, and pericardial space. Chest. 2013;144(5):1735-6.

14. Trovato GM, Sperandeo M, Catalano D. Optimization of thoracic US guidance for lung nodule biopsy. Radiology. 2014;270(1):308.

15. Trovato GM, Sperandeo M, Catalano D. Computed tomography screening for lung cancer. Ann Intern Med. 2013;159(2):155.

16. Sperandeo M, Rotondo A, Guglielmi G, Catalano D, Feragalli $B$, Trovato GM. Transthoracic ultrasound in the assessment of pleural and pulmonary diseases: use and limitations. Radiol Med. 2014 Feb 5.

17. Catalano D, Sperandeo M, Sacco MC, Trovato G. Lung ultrasound in pediatric pneumonia. The persistent need of chest X-rays. Pediatr Pulmonol. 2013 Oct 31.

18. Bolliger CT, Herth FJF, Mayo PH, Miyazawa T, Beamis JF. Clinical Chest Ultrasound. Prog Resp Res. 2009;37:1-221.

19. Pozniak MA, Zagzebski JA, Scanlan KA. Spectral and color Doppler artifacts. Radiographics. 1992; 12(1):35-44.

20. Eibenberger KL, Dock WI, Ammann ME, Dorffner R, Hörmann MF, Grabenwöger F. Quantification of pleural effusions: sonography versus radiography. Radiology.1994;191(3):681-4.

21. Sperandeo M, Caturelli E, Sperandeo G, Camagna
A. A new technique of thoracentesis in massive $\mathrm{Hy}$ drothorax. J Hepatol 2002;36 (Supplement)1:209.

22. Targhetta R, Bourgeois JM, Balmes P. Echography of pneumothorax. Rev Mal Respir. 1990;7(6):575-9.

23. Wernecke K, Galanski M, Peters PE, Hansen J. Pneumothorax: evaluation by ultrasound-preliminary results. J Thorac Imaging. 1987;2(2):76-8.

24. Lim JH, Lee KS, Kim TS, Chung MP. Ring-down artifacts posterior to the right hemidiaphragm on abdominal sonography: sign of pulmonary parenchymal abnormalities. J Ultrasound Med. 1999;18(6):403-10.

25. Sperandeo M, Varriale A, Sperandeo G, Bianco MR, Piattelli ML, Bizzarri M, et al. Characterization of the normal pulmonary surface and pneumonectomy space by reflected ultrasound. J Ultrasound. 2011;14 (1):22-7.

26. Sperandeo M, Filabozzi P, Varriale A, Carnevale V, Piattelli ML, Sperandeo G, et al. Role of thoracic ultrasound in the assessment of pleural and pulmonary diseases. JUS 2008;11(2):39-46.

27. Sargsyan AE, Hamilton DR, Nicolaou S, Kirkpatrick AW, Campbell MR, Billica RD, et al. Ultrasound evaluation of the magnitude of pneumothorax: a new concept. Am Surg. 2001;67(3):232-5.

28. Yang PC, Luh KT, Chang DB, Wu HD, Yu CJ, Kuo $\mathrm{SH}$. Value of sonography in determining the nature of pleural effusion: analysis of 320 cases. AJR Am J Roentgenol. 1992;159(1):29-33.

29. Görg C, Görg K, Schwerk WB, Kleinsorge F.Sonography of the diaphragmatic pleura in tumor patients. Ultraschall Med. 1988;9(6):274-8.

30. Kohan JM, Poe RH, Israel RH, Kennedy JD, Benazzi $\mathrm{RB}$, Kallay MC,et al. Value of chest ultrasonography versus decubitus roentgenography for thoracentesis. Am Rev Respir Dis. 1986;133(6):1124-6.

31. Pihlajamaa K, Bode MK, Puumalainen T, Lehtimäki A, Marjelund S, Tikkakoski T. Pneumothorax and the value of chest radiography after ultrasound-guided thoracocentesis. Acta Radiol. 2004;45(8):828-32.

32. Prasad V, Gall V, Cifu A. The frequency of medical reversal. Arch Intern Med. 2011;171(18):1675-6.

33. Prasad V, Vandross A, Toomey C, Cheung M, Rho J, Quinn S,et al. A decade of reversal: an analysis of 146 contradicted medical practices. Mayo Clin Proc. 2013;88(8):790-8. 